\title{
Role of the Ratio of Trans Cerebellar Diameter and Abdominal Circumference in Detecting Asymmetrical Intra Uterine Growth Restriction
}

\author{
Joydeep Roy ${ }^{1}$, Poushali Sanyal ${ }^{2}$, Varsha Saboo ${ }^{3}$, Raju Gopal Saha ${ }^{4}$, Sarumathy K. A. ${ }^{5}$, Rituparna Raj ${ }^{6}$, Chaitali DuttaRay ${ }^{7}$ \\ ${ }^{1}$ Department of Obstetrics and Gynaecology, IPGME\&R, Kolkata, West Bengal, India. ${ }^{2}$ Department of Obstetrics and \\ Gynaecology, IPGME\&R, Kolkata, West Bengal, India. ${ }^{3}$ Department of Obstetrics and Gynaecology, IPGME\&R, Kolkata, \\ West Bengal, India. ${ }^{4}$ Department of Obstetrics and Gynaecology, Burdwan Medical College, Burdwan, West Bengal, \\ India. ${ }^{5}$ Department of Obstetrics and Gynaecology, IPGME\&R, Kolkata, West Bengal, India. ${ }^{6}$ Department of Obstetrics \\ and Gynaecology, IPGME\&R, Kolkata, West Bengal, India. ${ }^{7}$ Department of Obstetrics and Gynaecology, IPGME\&R, \\ Kolkata, West Bengal, India.
}

\section{ABSTRACT}

\section{BACKGROUND}

Antenatal ultrasound of the foetus has brought considerable improvements in antenatal care without resultant major complications. It helps a lot in timely and accurate recognition of intrauterine growth restriction, which has a 10\% incidence in the general population. This study was carried out to determine a statistically significant cut-off value or range for the ratio of Trans Cerebellar Diameter (TCD)/Abdominal Circumference (AC) by which foetal growth restriction can be diagnosed after 28 weeks of gestation. The study aimed at evaluating the accuracy of TCD measured by ultrasound in predicting the gestational age and asymmetric IUGR prenatally.

\section{METHODS}

The study population comprised of antenatal mothers attending the Gynaecology and Obstetrics OPD and indoor clinic at IPGMER and SSKM Hospital over a period of 1 year. An estimated 100 mothers constituted the study subjects- 50 clinically suspected IUGR pregnancies and 50 presumably normal pregnancies. It was an observational analytical study. No separate control group was required. Babies without IUGR within the study cohort served as control. After obtaining history and examination, ultrasonic measurements on the subjects were made with 2dimensional real time ultrasound. Electronic callipers were used to measure TCD in outer to outer fashion.

\section{RESULTS}

Fetal parameters like BPD, AC, FL have good sensitivity in assessing IUGR as their values are significantly different from normal pregnancies. Head circumference and TCD values do not change significantly between age matched IUGR foetuses. TCD has linear variation with gestational age and thus is a better parameter for assessing gestational age than other ones.

\section{CONCLUSIONS}

The TCD/AC ratio, which utilizes both the least and the most affected foetal biometric parameters, should provide a very sensitive method for detecting asymmetrical and possibly symmetrical IUGR at any gestational age. This ratio may be especially useful in the evaluation of patients with poor or unknown gestational dating, allowing for early clinical intervention in abnormal cases.

\section{KEY WORDS}

Ratio, Trans Cerebellar Diameter, Abdominal Circumference, Intrauterine Growth Restriction
Corresponding Author: Poushali Sanyal,

\#192/A,

Shyama Prasad Mukherjee Road,

Kolkata-700026,

West Bengal, India.

E-mail: poushali.sanyal@yahoo.co.in

DOI: $10.14260 / \mathrm{jemds} / 2019 / 836$

Financial or Other Competing Interests: None.

How to Cite This Article:

Roy J, Sanyal P, Saboo V, et al. Role of the ratio of trans cerebellar diameter and abdominal circumference in detecting asymmetrical intra uterine growth restriction. J. Evolution Med. Dent. Sci. 2019;8(51):3858-3862, $10.14260 / j e m d s / 2019 / 836$

Submission 10-09-2019,

Peer Review 17-11-2019,

Acceptance 23-11-2019,

Published 23-12-2019.

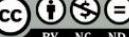




\section{BACKGROUND}

The incidence of intrauterine growth restriction (IUGR) in general obstetric population is high $(\sim 10 \%)$ but its recognition is low ( $<40 \%)$. Regular surveillance and timely management decisions are the cornerstones for optimum outcome in these cases, which may rely mainly on accurate determination of gestational age to avoid increase in perinatal morbidity and mortality. ${ }^{1}$ Antenatal ultrasound of the foetus has brought considerable improvements in antenatal care without resultant major complications. It offers help in pregnancies where date of conception is uncertain. Ultrasonography is often the initial imaging method used to evaluate the foetus and monitor foetal growth. It can be performed easily and non-invasively with no risk to foetus, mother, or operator. Many foetal parameters can be measured by ultrasonography and have been correlated with gestational age.

Usefulness of these biometric parameters in establishing gestational age in the third trimester of pregnancy or assessing foetal growth in pregnancies with uncertain dating is limited. In most of such cases, serial ultrasonography evaluation is required to differentiate normal from abnormal foetal growth. So there is a need for reliable parameters for ultrasound dating, especially in patients with wrong dates or unknown dates with late antenatal registration and also in those compounded with foetal growth restriction ${ }^{2}$. Trans cerebellar Diameter (TCD), a measurement in the posterior fossa of foetal brain is least affected by external factors and foetal growth abnormalities, which allows its use for gestational age determination even in third trimester ${ }^{3}$. Instead, foetal Abdominal Circumference (AC) is one the important foetal parameters to be affected early in the process of impaired foetal growth. So, Trans cerebellar Diameter/Abdominal Circumference (TCD/AC) ratio has been found to be constant and a gestational age independent parameter to assess foetal growth and can be useful in predicting foetal growth restriction. ${ }^{4}$ Hence, the study was carried out to determine a statistically significant cut-off value or range for the ratio of Trans cerebellar Diameter (TC)/Abdominal Circumference (AC) by which foetal growth restriction can be diagnosed after 28 weeks of gestation.

\section{Aim}

To evaluate the accuracy of Trans cerebellar diameter in predicting the gestational age and asymmetric IUGR prenatally.

\section{Objectives}

1. To assess any correlation between gestational age and TC Diameter measurement.

2. To assess whether IUGR detection is possible by measuring TCD/AC prenatally.

3. To determine a statistically significant cut-off value/range for the ratio of TCD/AC by which IUGR can be diagnosed after 28 weeks of gestation.

4. To assess maternal and prenatal outcome in foetuses diagnosed with growth restriction using this parameter.

\section{METHODS}

The study population comprised antenatal mothers attending the Gynaecology and Obstetrics OPD and indoor clinic at IPGMER and SSKM Hospital These patients came from in and around Kolkata. Since this is a tertiary referral centre, patients also came from rural areas. The study was conducted over a period of 1 Year from August 2016 to July 2017. An estimated 100 mothers constituted the study subject - 50 clinically suspected IUGR pregnancies and 50 presumably normal pregnancies. The sample size was determined by statistical calculation using SPSS software. It was an observational analytical study. No separate control group was required. Babies without IUGR within the study cohort served as control. IUGR was determined by standard definition if the AC or Estimated foetal weight was less than the $10^{\text {th }}$ centile for that gestational age.

\section{Inclusion Criteria}

All singleton, non-anomalous pregnancies beyond 20 weeks of gestation presenting with cephalic presentation.

\section{Exclusion Criteria}

- Non-Cephalic Presentation

- Multiple Gestation.

- Anomalous Pregnancies.

- Moderate to severe polyhydramnios.

- Previous irregular or unknown menstrual history with no dating scan.

- Maternal age $<18$ years and $>35$ years.

- $\quad$ Symmetrical IUGR (after USG).

\section{Study Tools}

1. The following Laboratory Investigations were done: Routine Blood Investigations (including counting Blood Count, ABO Grouping and Rh typing, HBsAg, VDRL, ICTC, Thalassemia screening, FBS/PPBS, Thyroid Profile) were carried out.

2. Transabdominal Sonography was done with the USG machine made by TOSHIBA, MODEL XARIO, using TAS curvilinear probe of frequency range $2.5-5 \mathrm{MHz}$. The following scans were done during the antenatal period:

- $\quad$ 1st Trimester dating scan (7-10 weeks)

- Anomaly scan at 18 to 20 weeks of gestation

- Pregnancy profile at 28- 34 weeks of gestation including Trans cerebellar diameter/Abdominal Circumference ratio determination

- Pregnancy profile at 36-40 weeks of gestation including TCD/AC ratio determination (if needed)

\section{Study Technique}

After taking detailed history, performing clinical examinations and routine investigations, patients with the above-mentioned exclusion criteria were excluded. Informed consent was taken from the rest for their voluntary participation in the study after proper counselling about the procedure. 


\section{Real Time Ultrasonography}

The ultrasonic measurements were made with 2-dimensional real time ultrasound. The machine was made by Toshiba, and the name of the model was Toshiba Xario 100. The transducer used was a curvilinear transducer of frequency of 3.5 Mega Hertz, and the model was PVT 375 BT.

Guidelines for Measuring the Trans Cerebellar Diameter The cerebellum is located in the posterior fossa and visualized by slight posterior and inferior rotation of the transducer at the level of BPD. Electronic callipers were used to measure TCD in outer to outer fashion as described by Goldstone et al. ${ }^{5}$

\section{Statistical Analysis}

Data was summarised by routine descriptive statistics, namely mean, and standard deviation for normally distributed numerical variables, median and interquartile range for skewed numerical variables and counts and percentages for categorical variables. The predictive ability of TCD: AC ratio at 28 to 34 weeks was assessed in terms of standard diagnostic indices (sensitivity, specificity, positive predictive value, negative predictive value), and also kappa statistics. An attempt was made to determine the optimum cut-off of the said ratio in predicting IUGR through receiver operating characteristics (ROC) curve analysis.

\section{RESULTS}

In our study, the mean age of female in normal pregnancies is $24.17 \pm 2.17$ years, in IUGR $27.37 \pm 3.56$ years. There is a significant difference between ages of these groups showing that more aged females are more prone to develop IUGR foetus. In this study, prevalence of medical complications is $13 \%$. Amongst which, history of infertility is in $5 \%$, Hypothyroid in $6 \%$ and heart disease in $2 \%$. There is no correlation between the medical complication and incidence of IUGR. Table 1 shows the foetometric data of normal pregnancy. All the parameters increase with age of foetus except TCD/AC ratio which remains constant. Table 2 shows the foetometric data of IUGR pregnancies. All the parameters increase with age of foetus including TCD/AC value.

Table 3 suggests that foetal parameters like BPD, AC, FL have good sensitivity in assessing IUGR as their values are significantly different from normal pregnancies. Head circumference and TCD values doesn't change significantly between ages matched IUGR foetuses. Ratio of TCD/AC is significantly different between two groups showing that though TCD values remain almost constant between two groups, it's only AC which changes between normal and IUGR categories, thus increasing the value of the ratio. It was seen that 25 cases were below the fifth percentile for BPD and FL, 22 cases were below the fifth percentile for AC, 9 cases were below the fifth percentile for HC. In 21 out of 29 cases the TCD values were within the normal range. Only in 8 cases the TCD values were below the 5 th percentile. This difference in number of cases below the 5 th percentile for BPD, HC, AC, FL and TCD was statistically significant ( $p$-value $=0.001$ ).

In 29 IUGR cases the gestational age predicted by transverse cerebellar diameter measurements closely correlated with gestational age predicted by last menstrual period. Though the gestational age predicted by BPD, HC, AC and FL also has good sensitivity in 18-20 weeks age group. 18 out of 29 IUGR have TCD/AC ratio above 2SD, showing that the ratio is higher in majority of IUGR cases of in 18-20 weeks age group. The table no. 4 suggests that values of SFH, BPD, AC and TCD/AC are significantly different between two groups. It was seen that 21 cases for BPD and 9 cases for FL were below the fifth percentile, 22 cases were below the fifth percentile for AC, 5 cases were below the fifth percentile for HC. In 29 out of 29 cases the TCD values were within the normal range showing the TCD values doesn't change in IUGR pregnancies.

The delivery outcomes in this group is shown in Table 5. $\mathrm{BPD}$ and $\mathrm{AC}$ proved to have good sensitivity in IUGR prediction while FL independently has poor diagnostic accuracy. In 29 IUGR cases the gestational age predicted by transverse cerebellar diameter measurements closely correlated with gestational age predicted by last menstrual period. In 29 out of 29 IUGR, TCD/AC ratio remains above 2SD, showing that the ratio is higher in all the IUGR cases in 28-34 weeks age group. From Table 6 it can be deduced that out of the total 29 confirmed IUGR cases, TCD/AC ratio could predict the asymmetric growth retardation in $100 \%$ of growth retarded, but also showed false positive prediction in 4 cases. So the sensitivity of TCD/AC in IUGR prediction is $100 \%$ while in specificity is $80.95 \%$. The distribution in above mentioned scatter diagram shows that TCD has linear variation with gestational age and thus is a better parameter for assessing gestational age than other ones.

\begin{tabular}{|c|c|c|c|c|c|c|c|c|}
\hline $\begin{array}{c}\text { Foetal } \\
\text { Age }\end{array}$ & $\begin{array}{l}\text { SFH } \\
(\mathrm{cm})\end{array}$ & BPD & AC & FL & HC & TCD & $\begin{array}{c}\text { TCD } / \\
\text { AC }\end{array}$ & EFW \\
\hline $18-20$ & $\begin{array}{c}17.14 \pm \\
1.27\end{array}$ & $\begin{array}{c}48.53 \pm \\
2.34\end{array}$ & $\begin{array}{l}46.75 \pm \\
15.49\end{array}$ & & & & \begin{tabular}{|c|}
$0.13 \pm$ \\
0.01 \\
\end{tabular} & \\
\hline $28-34$ & $\begin{array}{c}29.14 \pm \\
2.19\end{array}$ & $\begin{array}{c}78.48 \pm \\
3.75\end{array}$ & $\begin{array}{c}253.79 \pm \\
29.45\end{array}$ & $\begin{array}{c}56.8 \pm \\
4.57\end{array}$ & $\begin{array}{c}278.39 \pm \\
16.89\end{array}$ & $\begin{array}{c}33.78 \pm \\
3.85\end{array}$ & 0.13 & $\begin{array}{c}1488.78 \pm \\
335.6\end{array}$ \\
\hline $36-40$ & $\begin{array}{c}32.95 \pm \\
2.44\end{array}$ & $\begin{array}{c}84.36 \pm \\
4.34\end{array}$ & $\begin{array}{c}290.08 \pm \\
78.32 \\
\end{array}$ & $\begin{array}{c}63.19 \pm \\
4.58\end{array}$ & $\begin{array}{c}316.60 \pm \\
17.42 \\
\end{array}$ & $\begin{array}{c}39.60 \pm \\
8.65\end{array}$ & 0.134 & $\begin{array}{c}2704.07 \pm \\
239.6 \\
\end{array}$ \\
\hline \multicolumn{9}{|c|}{ Table 1. Foetometric Data of Normal Pregnan } \\
\hline
\end{tabular}

\begin{tabular}{|c|c|c|c|c|c|c|c|c|}
\hline $\begin{array}{c}\text { Foetal } \\
\text { Age }\end{array}$ & $\begin{array}{c}\text { SFH } \\
\text { (cm) }\end{array}$ & BPD & AC & FL & HC & TCD & $\begin{array}{c}\text { TCD/ } \\
\text { AC }\end{array}$ & EFW \\
\hline \multirow{2}{*}{$18-20$} & $\begin{array}{c}17.27 \pm \\
0.86\end{array}$ & $\begin{array}{c}41.24 \\
\pm 5.12\end{array}$ & $\begin{array}{c}123.34 \pm \\
12\end{array}$ & $\begin{array}{c}34.65 \pm \\
6.41\end{array}$ & $\begin{array}{c}145 \pm \\
14.23\end{array}$ & $\begin{array}{c}17.24 \pm \\
1.13\end{array}$ & $\begin{array}{c}0.14 \pm \\
0.01\end{array}$ & $331.96 \pm$ \\
& Table 2. Foetometric Data of IUGR Pregnancies \\
\hline $28-34$ & $\begin{array}{c}23.62 \pm \\
1.78\end{array}$ & $\begin{array}{c}71.17 \\
\pm 5.96\end{array}$ & $\begin{array}{c}244.37 \\
\pm 13\end{array}$ & $\begin{array}{c}56.55 \pm \\
5.33\end{array}$ & $\begin{array}{c}272.24 \pm \\
11.46\end{array}$ & $\begin{array}{c}38.59 \pm \\
2.74\end{array}$ & 0.158 & $\begin{array}{c}1381.79 \pm \\
237.4\end{array}$ \\
\hline \multirow{2}{*}{$36-40$} & $\begin{array}{c}30.07 \pm \\
1.51\end{array}$ & $\begin{array}{c}81.55 \pm \\
4.16\end{array}$ & $\begin{array}{c}238.51 \pm \\
32.65\end{array}$ & $\begin{array}{c}65.88 \pm \\
4.379\end{array}$ & $\begin{array}{c}320.44 \pm \\
22.13\end{array}$ & $\begin{array}{c}38.25 \pm \\
5.07\end{array}$ & $\begin{array}{c}0.16 \pm \\
0.09\end{array}$ & $\begin{array}{c}2229.7 \pm \\
53.51\end{array}$ \\
\hline
\end{tabular}

\begin{tabular}{|c|c|c|c|}
\hline Foetal Parameters & Normal & IUGR & 'p' Value \\
\hline SFH & $17.14 \pm 1.27$ & $17.27 \pm 0.86$ & 0.633 \\
\hline BPD & $48.53 \pm 2.34$ & $41.24 \pm 5.12$ & 0.000 \\
\hline AC & $146.75 \pm 15.49$ & $123.34 \pm 12$ & 0.000 \\
\hline FL & $33.7 \pm 3.16$ & $28.06 \pm 2.77$ & 0.015 \\
\hline HC & $168.09 \pm 12.16$ & $160.03 \pm 14.36$ & 0.09 \\
\hline TCD & $19.10 \pm 0.99$ & $18.18 \pm 0.99$ & 0.08 \\
\hline TCD/AC & $0.13 \pm .01$ & $0.14 \pm 0.01$ & 0.008 \\
\hline EFW & $386.63 \pm 58.66$ & $331.96 \pm 36.41$ & 0.004 \\
\hline \multicolumn{4}{|l}{ Table 3. Comparison of 18-20 Weeks Foetometry } \\
\hline
\end{tabular}

\begin{tabular}{|c|c|c|c|}
\hline Foetal Parameters & Normal & IUGR & 'p' Value \\
\hline SFH & $29.14 \pm 2.19$ & $23.62 \pm 1.78$ & 0.000 \\
\hline BPD & $78.48 \pm 3.75$ & $71.17 \pm 5.96$ & 0.000 \\
\hline AC & $253.79 \pm 29.45$ & $223.41 \pm 12.55$ & 0.000 \\
\hline FL & $56.8 \pm 4.57$ & $54.62 \pm 4.90$ & 0.689 \\
\hline HC & $278.39 \pm 16.89$ & $272.24 \pm 11.46$ & 0.060 \\
\hline TCD & $33.78 \pm 3.85$ & $35.25 \pm 2.47$ & 0.029 \\
\hline TCD/AC & $0.13 \pm 0.009$ & 0.158 & 0.000 \\
\hline EFW & $1488.78 \pm 335.6$ & $1381.79 \pm 237.4$ & 0.732 \\
\hline
\end{tabular}

Table 4. Comparison of Foetometric Values in 28-34 Weeks Pregnancy 


\begin{tabular}{|c|c|c|c|}
\hline \multicolumn{4}{|c|}{ Delivery Outcome } \\
\hline USG (TCD/AC) & IUGR + & IUGR - & \\
\hline IUGR + & $29($ True +$)(a)$ & $4($ False +$)(c)$ & 33 (All test + ) \\
\hline IUGR - & $0($ False -$)(\mathrm{b})$ & $17($ True -$)(\mathrm{d})$ & 17 (All test - ) \\
\hline & $a+b=29$ & $c+d=21$ & 50 \\
\hline
\end{tabular}

\begin{tabular}{|c|c|c|}
\hline Sensitivity & $100 \%$ & $\mathrm{a} /(\mathrm{a}+\mathrm{b})$ \\
\hline Specificity & $80.95 \%$ & $\mathrm{~d} /(\mathrm{c}+\mathrm{d})$ \\
\hline Positive Predictive Value & $87.88 \%$ & $\mathrm{a} /(\mathrm{a}+\mathrm{c})$ \\
\hline Negative Predictive Value & $100 \%$ & $\mathrm{~d} /(\mathrm{b}+\mathrm{d})$ \\
\hline \multicolumn{2}{|c|}{ Table 6. Diagnostic Accuracy Variables for TCD/AC Ratio } \\
between 28-34 Weeks \\
\hline
\end{tabular}

\section{DISCUSSION}

Accurate gestational dating is of paramount importance and cornerstone for management of pregnancies especially those with foetuses who have growth disturbances (IUGR foetuses). In the present study, there was good correlation between TCD and gestational age ( $\mathrm{r} 2=0.975, \mathrm{p}$-value $=0.001)$. Also, good correlation was found between TCD and BPD ( $\mathrm{r} 2=0.976$, $\mathrm{p}$ value $=0.001)$ and between TCD and HC (r2=0.976, pvalue $=0.001$ ). In this study TCD nomogram was established from USG measured data which can be used for estimating the gestational age of foetus. Similar results were obtained in the present study. We noticed a curvilinear relationship between TCD and gestational age ( $r 2=0.992, p$-value $=0.004)$. In all the 50 IUGR cases TCD showed good correlation with known gestational age. These results were similar to the study by Reece et al. ${ }^{6}$

The TCD/AC ratio, which utilizes both the least and the most affected foetal biometric parameters, should provide a very sensitive method of detecting asymmetrical and possibly symmetrical IUGR at any gestational age. This ratio may be especially useful in the evaluation of patients with poor or unknown gestational dating, allowing for early clinical intervention in abnormal cases.

The foetal cerebellum is easily visualized in the posterior fossa from approximately 11 weeks onward. ${ }^{4}$ In its location between the dense petrous ridges, the cerebellum is protected from external compressive forces that may distort the foetal cranium, as seen with breech presentation or oligohydramnios. In these circumstances, measurements of the BPD and HC will be less accurate estimators of gestational age. Because of its protected location, the correlation of TCD with gestational age is preserved in these cases and is better than that of the BPD and HC.

Haller et al., studied 635 well-dated, normal pregnancies and demonstrated a strong correlation between GA determined by LMP and both foetal TCD (r2=0.91338) and AC $(\mathrm{r} 2=0.89361)$ in foetuses with birth weights between the $10^{\text {th }}$ and $90^{\text {th }}$ percentiles. In spite of poor correlation of TCD/AC ratio with gestational age ( $\mathrm{r} 2=0.15788$ ), a slight increase with TCD/AC ratio > 15.5 was present in $80 \%$ of SGA infants with increasing gestation. ${ }^{7}$

In this study the mean value of TCD/AC ratio in control group was $13 \pm 0.90$ and in patients with confirmed asymmetric growth retardation on post-delivery examination was $0.160 \pm 0.09$, significantly higher $(\mathrm{p}<0.05)$ than in postdelivery normal cases $(\mathrm{p}<0.05)$ In this study, out of 29 true IUGR cases were correctly diagnosed by TCD/AC ratio, hence sensitivity was $88.89 \%$, specificity $85.71 \%$, the PPV was $88.89 \%$ and NPV was $85.71 \%$. These results corroborated well with a similar study by Tonsong et al. ${ }^{8}$

In this study, three IUGR pregnancies delivered by LSCS while one had instrumental delivery. Amongst those IUGR pregnancies, 3 had preterm delivery. While amongst those pregnancies who were predicted as IUGR but confirmed not to be IUGR by TCD/AC ratio, only 1 delivered by LSCS and 1 by instrumental delivery. There were no preterm deliveries in this group. Thus, we can conclude that TCD/AC ratio could predict perinatal and fatal outcome to a certain extent. Those who have TCD/AC value more than 2SD of cut off value have higher chances of adverse perinatal and foetal outcome.

One of the important limitations in the ultrasound study is dependency on operator for precise measurement. In the present study measurements were taken by different operators as many reports had to be traced retrospectively thus increasing inter operator variability. With prospective as well as retrospective study design, the possibility of inherent bias for the radiologist while measuring the foetal biometric parameters increased in this study. Another technical limitation is the dense shadowing in posterior fossa in third trimester, which may limit adequate visualisation of cerebellum.

\section{CONCLUSIONS}

There is good correlation between TCD and other parameters in normal pregnancies at 15 to 40 weeks of gestation. TCD is a better parameter for gestational age assessment compared to BPD, AC and FL. TCD measurements are not affected by conditions which affect other foetometric parameters. TCD can be used to predict the gestational age in IUGR pregnancies as it correlates with gestational age more closely than other parameters. Ratio of TCD/AC remains constant throughout the normal pregnancy; thus, we could find out a cut off value which is $13 \pm 0.90$, which proved to have a sensitivity of $100 \%$ and specificity of $80.95 \%$ in predicting IUGR.

\section{REFERENCES}

[1] Peleg D, Kennedy CM, Hunter SK. Intrauterine growth restriction: identification and management. Am Fam Physician 1998;58(2):453-60, 466-7.

[2] Lee W, Barton S, Comstock CH, et al. Transverse cerebellar diameter: a useful predictor of gestational age for foetuses with asymmetric growth retardation. Am J Obstet \& Gynecol 1991;165(4 Pt 1):1044-50.

[3] Campbell WA, Vintzileos AM, Rodis JF, et al. Use of the transverse cerebellar diameter/abdominal circumference ratio in pregnancies at risk for intrauterine growth retardation. J Clin Ultrasound 1994;22(8):497502.

[4] Degani S. foetal biometry: Clinical, pathological and technical considerations. Obstet Gynaecol Surv 2001;56(3):159-67. 
[5] Goldstein I, Reece EA, Pilu G, et al. Cerebellar measurements with ultrasonography in the evaluation of foetal growth and development. Am J Obstet Gynecol 1987;156(5):1065-9.

[6] Reece EA, Goldstein I, Pilu G, et al. foetal cerebellar growth unaffected by intrauterine growth retardation: a new parameter for prenatal diagnosis. Am J Obstet Gynecol 1987;157(3):632-8.
[7] Sharma C, Bhardwaj A, Kharkwal S. foetal trans cerebellar diameter measurement for prediction of gestational age: more dependable parameter even in IUGR. International Journal of Gynae Plastic Surgery 2014;6(1):13-8.

[8] Tonsong T, Wanapirak C, Thongpadungrogj T. Sonographic diagnosis of intrauterine growth restriction by foetal transverse cerebellar diameter to abdominal circumference ratio. Int J Gynecol Obstet 1999;66(1):1-5. 